Rabaska

Revue d'ethnologie de l'Amérique française

\title{
Dean Louder (1943-2017)
}

\section{Yves Frenette et André Fauchon}

Volume 15, 2017

URI : https://id.erudit.org/iderudit/1041132ar

DOI : https://doi.org/10.7202/1041132ar

Aller au sommaire du numéro

Éditeur(s)

Société québécoise d'ethnologie

ISSN

1703-7433 (imprimé)

1916-7350 (numérique)

Découvrir la revue

Citer ce document

Frenette, Y. \& Fauchon, A. (2017). Dean Louder (1943-2017). Rabaska, 15, 207-209. https://doi.org/10.7202/1041132ar d'utilisation que vous pouvez consulter en ligne.

https://apropos.erudit.org/fr/usagers/politique-dutilisation/ 


\section{Dean Louder (1943-2017)}

Dean Louder n'était pas poète, mais nous, ses amis, ses collègues, ses anciens étudiants, sommes nombreux à le pleurer depuis son décès, survenu le 9 mai 2017 à l'âge de 74 ans.

Dean est né à Park City, dans l'Utah. Son aventure dans la francophonie commence à dix-neuf ans, lors d'un séjour de deux ans à Paris, non pas pour y goûter les plaisirs de la vie parisienne, mais comme missionnaire mormon. Il fait ensuite ses études universitaires à Salt Lake City, puis à l'University of Washington (Seattle). C'est là qu'il fait la connaissance de Paul Villeneuve, doctorant comme lui. Le jeune géographe québécois fait

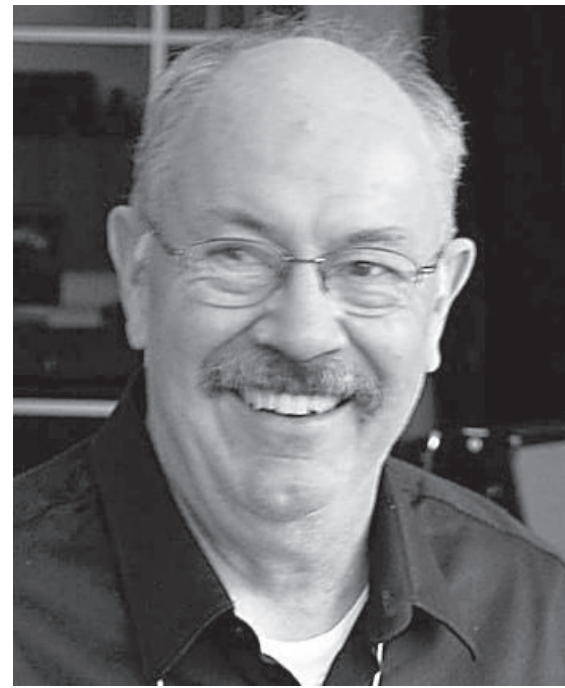

Quand il est mort, le poète, Tous ses amis pleuraient

Louis Amade connaître Dean au Département de géographie de l'Université Laval, où on cherche ardemment des recrues pour enseigner cette nouvelle géo quantitative à laquelle tous deux ont été formés à Seattle.

Dean arrive à Laval en 1971. Il y enseigne la géographie sociale. Il s'intéresse à l'espace des minorités - enfants, personnes âgées, Noirs, populations démunies - notamment aux États-Unis. En 1977-1978, il passe sa première année sabbatique en Louisiane, pays de son épouse Billie. Il y retrouve l'Amérique, non pas celle de son enfance ou de son enseignement, mais une Amérique différente, une Amérique française, qu'il découvre au gré du projet de recherche "Louisiane », où il œuvre en compagnie d'anthropologues. Cette découverte orientera sa carrière, en faisant de lui un praticien de la géographie culturelle et un pionnier de l'étude des divers aspects de la francophonie nord-américaine. À la suite d'un colloque à la 
Nouvelle-Orléans, Dean codirige, en 1979, deux numéros thématiques de la revue Cahiers de géographie du Québec puis, en 1983, un ouvrage marquant, un ouvrage fondateur, Du continent perdu à l'archipel retrouvé : le Québec et l'Amérique française.

Que de chemins parcourus par la suite à sillonner de long en large et à maintes reprises les espaces francophones du continent : l'Acadie, la Louisiane, la Nouvelle-Angleterre, l'Ontario, le centre-ouest des ÉtatsUnis, l'Ouest canadien et américain, et une kyrielle de petites communautés francophones, de langue ou de mémoire, que peu de personnes connaissaient. Son enthousiasme était contagieux. Il a ainsi fait prendre conscience de l'existence de la francophonie nord-américaine à de nombreux étudiants des trois cycles universitaires, dans le cadre de cours et d'excursions que lui et son complice Éric Waddell organisèrent pendant un quart de siècle dans tous les coins et recoins de la Franco-Amérique, terme qu'ils créèrent à l'orée $\mathrm{du} \mathrm{XXI}^{\mathrm{e}}$ siècle. À chaque fois, ils sollicitaient des collègues spécialistes des milieux qu'ils visitaient et de nombreux artisans de la francophonie locale. La gageure, c'était pour les participants de comprendre " par osmose ».

Dean avait des amis partout. On peut constater l'étendue de son réseau dans les ouvrages collectifs qu'il a codirigés. Ces livres ont ceci d'unique qu'ils donnent la parole à celles et ceux dont ils ont étudié la destinée. FrancoAmérique, paru originellement en 2008 et réédité en 2017, en témoigne : s'y retrouvent côte à côte universitaires, romanciers, cinéastes, leaders communautaires, etc. Ou encore mieux Vision et visages de la FrancoAmérique, publié en 2001, dans lequel chercheurs et intellectuels cohabitent avec de « parfaits inconnus ».

À partir de sa retraite, en 2003, Dean reprend, le plus souvent en solitaire, ses « arpentages » de la Franco-Amérique, mais beaucoup plus lentement, « au rythme de l'escargot». Il diffuse ses carnets de voyage, d'abord sur le site du Conseil de la vie française en Amérique, puis sur celui des Éditions du Septentrion. La majorité de ces chroniques sont réunies, dix ans plus tard, dans le livre Voyages et rencontres en Franco-Amérique.

Arrivés au Québec en pleine effervescence nationaliste, Dean Louder et Éric Waddell firent leur la quête de pays d'un grand nombre de Québécois. Par ricochet, l'Amérique française qu'ils décrivirent et définirent au début des années 1980 était essentiellement laurentienne, bien qu'ils fissent une certaine place à l'Acadie et à la Louisiane. Leur thèse était la suivante : au XVIII ${ }^{\mathrm{e}}$ siècle, à partir de la vallée du Saint-Laurent, les Canadiens français se sont répandus sur tout le continent, s'établissant en différents lieux qui en vinrent à former un ensemble, l'Amérique française. Par la suite, cet ensemble se fragmenta en îlots. Cependant, à la fin du Xx ${ }^{\mathrm{e}}$ siècle, il retrouvait une certaine unité, le Québec jouant le rôle de véritable Mecque pour les descendants, 
souvent assimilés, des expatriés. Ce n'était donc plus la langue qui cimentait l'Amérique française de Louder et Waddell, mais une façon d'être s'abreuvant à des réseaux plutôt flous et à une mémoire des origines. Bien qu'ils s'en fussent certainement défendus, les deux géographes promouvaient alors une version remaniée du messianisme canadien-français de jadis, moins clérical, plus poétique. Cette position eut l'heur d'agacer des chercheurs, tant québécois que canadiens, américains et français. Toutefois, à force de pérégriner et sous l'influence de certains de leurs disciples, et aussi de leurs critiques, Louder et Waddell prirent conscience de la diversité franco-américaine, au sens large du terme, du poids culturel des Antilles dans la constitution de la francophonie nord-américaine et de l'existence de la créolité. C'est de cette manière que, au tournant du XXI ${ }^{\mathrm{e}}$ siècle, leur Amérique française devint une Franco-Amérique.

Dean se démarquait par ses qualités humaines. Père de huit enfants et grand-père de vingt petits-enfants, il montrait un grand respect, une grande empathie pour les gens qu'il rencontrait, toujours à l'affût de ce qu'ils avaient de meilleur à offrir à la vie française sur le continent. Jamais il ne travestit ce que ses interlocuteurs lui confiaient, laissant à ses auditoires et à ses lecteurs, pour lesquel il avait également un profond respect, le soin d'interpréter les propos dont il se voulait un simple relais. De témoin fidèle, il n'en est pas moins devenu un acteur important de la francophonie, ne cessant jamais de revendiquer la légitimité de cultures minoritaires négligées et oubliées dans l'ensemble continental anglo-américain. Francophone de langue maternelle anglaise, Dean avait non seulement adopté le français comme langue d'usage, mais était habité par le français. À une époque où le monde universitaire s'entichait de la publication en anglais, Dean choisit de faire paraître ses travaux en français.

C'est cet engagement total et profond envers la francophonie que reconnut le gouvernement du Québec en le faisant membre de l'Ordre des francophones d'Amérique (1996), le Centre de recherche en civilisation canadiennefrançaise de l'Université d'Ottawa en lui accordant son prestigieux Prix du CRCCF (conjointement avec Éric Waddell en 2008) et l'University of Manitoba en lui décernant un doctorat honorifique (2015)․․

Bon voyage, très cher Dean.

Yves Frenette et André Fauchon Université de Saint-Boniface

1. Les auteurs se sont permis d'emprunter librement au bel hommage rendu à Dean Louder et Éric Waddell par Anne Gilbert lors de leur obtention du Prix du CRCCF : arts.uottawa.ca/crecf/sites/arts. uottawa.ca.crcef/files/allocution_anne_gilbert_prixlouderwaddell.pdf. 